\title{
Equine-Assisted Psychotherapy: An Emerging Trauma-Informed Intervention
}

\author{
Page Walker Buck \\ Nadine Bean \\ Kristen de Marco
}

\begin{abstract}
Equine-assisted psychotherapy (EAP) has emerged as a promising intervention for the treatment of trauma and stressor-related disorders. This experiential therapy offers an option for clients whose traumatic experiences may render traditional talk therapies ineffective. Initial research on the most robust model of EAP, developed by the Equine Assisted Growth and Learning Association (EAGALA), indicates positive effects for children, adolescents, and adults who have experienced trauma. The EAGALA Model ${ }^{\circledR}$ was designed to allow for rigorous evaluation of efficacy, a clear theoretical base, standardized implementation, and ongoing training for practitioners. As the primary providers of mental and behavioral health services in the United States, social workers are keenly aware of the need for a portfolio of treatment methods to manage the increasing demand for services. This article provides an overview of EAP, including a review of the literature, the history of human-horse relations, an EAGALA case example, and a call for more rigorous research.
\end{abstract}

Keywords: Equine-assisted psychotherapy; trauma-informed; trauma and stressorrelated disorders; EAGALA

A number of cross-sectional and longitudinal studies regarding traumatic experiences indicate a far higher prevalence of exposure to trauma than once believed (Anda, Felitti, \& Corwin, 2014; Cronholm et al., 2015; Felitti et al., 1998; Murphy et al., 2016). Findings from the CDC-Kaiser Permanente Adverse Child Experiences (ACE) study reveal a graded dose-response relationship between ACEs and negative health and well-being outcomes across the lifespan (Anda et al., 2014). The more exposure an individual has to adverse events such as child maltreatment, intimate partner violence, violent crime, disaster, terrorism or war, the higher the likelihood of physical and mental health issues throughout the lifespan. Individuals face a higher risk of suffering from substance use disorders, depression, and trauma and stressor-related disorders, including post-traumatic stress disorder (PTSD). Unresolved trauma is linked to poor academic achievement, early sexual activity, financial stress, poor work performance, heart disease, and liver disease (Anda et al., 2014; Larkin, Shields, \& Anda, 2012).

The effects of trauma are significant, leaving indelible footprints on the brain. Increasingly, sensitive brain imaging techniques such as functional MRIs have illustrated that traumatic experiences, particularly severe and chronic exposure, affect brain development and functioning. Imaging results show changes in the amygdala, corpus callosum, cerebellum, hippocampus, and prefrontal cortex (Baker et al., 2013; Bourne,

Page Walker Buck, PhD, LSW and Nadine Bean, PhD, LCSW are Associate Professors in the Department of Graduate Social Work at West Chester University in West Chester, PA. Kristen de Marco is an EAGALA@-certified Equine Specialist and Executive Director of Gateway HorseWorks in Malvern, PA. 
Mackay, \& Holmes, 2013; Dannlowski et al., 2012). For individuals with a significant trauma history, these changes can overwhelm cognitive processes, including the ability to use words to express feelings and psychomotor sensations, as the prefrontal cortex goes off line (Sekiguchi et al., 2013; van der Kolk \& McFarlane, 2012).

In addition to the effects that trauma has on the brain, trauma also lives in the muscles, bones, and the neurons serving the musculoskeletal system (van der Kolk, 2014). van der Kolk (2014) explains that when the limbic system is repeatedly triggered during stress responses, there is an increased risk that it will remain on and leave the body in a hypervigilant state. Not surprisingly, traditional psychotherapy sessions can feel unsafe to clients whose systems are already overwhelmed. When the effects of trauma on the body are combined with the effects of trauma on cognitive processes, talk therapy alone is often rendered ineffective (van der Kolk, 2002; van der Kolk \& McFarlane, 2012). This is especially true for people who experienced their first traumatic experiences as children (Anda et al., 2006).

The Substance Abuse and Mental Health Services Administration (SAMHSA, 2014), in collaboration with the National Center for Trauma-Informed Care \& Alternatives to Seclusion and Restraint (NCTIC), outlines the Six Key Principles of a Trauma-informed Approach: 1) safety, 2) trustworthiness and transparency, 3) peer support, 4) collaboration and mutuality, 5) empowerment, voice and choice, and 6) cultural, historical and gender issues sensitivity. Underlying these principles is the conviction that recovery is possible and that through an appropriate trauma-informed approach; resiliency can be nurtured.

The notion of recovery is a shift in perspective from the medicalized use of the term disorder in describing post-traumatic stress response, particularly in the military and in disaster mental health services. As understanding about the nature of post-traumatic responses has evolved, there is a wider appreciation for the fact that trauma responses are normal reactions to abnormal situations (American Red Cross, 2012). The call to fill the treatment gap for complex trauma, however, is pressing. Practitioners need access to an expanded set of treatment options that includes modalities designed to specifically address the embodiment of trauma, especially given that the vast majority of mental and behavioral therapies are talk-based.

Rapidly emerging in the effort to enhance recovery and build resilience for trauma survivors are mind/body, experiential approaches and expressive therapies, including: mindfulness programs (Davis \& Hayes, 2011; Omidi, 2013), Trauma-focused Cognitive Behavioral Therapy or TF-CBT (Getz, 2012; Kliethermes \& Wamser, 2012), traumasensitive yoga (Rhodes, 2015; van der Kolk et al., 2014; West, Lian, \& Spinazzola, 2016), and expressive art therapies including psychodrama (Zucker, Spinazzola, Pollack, Pepe, \& Barry, 2010). By deemphasizing verbal communication, these approaches allow for the inclusion of non-verbal approaches that support the therapeutic relationship and the establishment of safety and trust, which in turn can improve affect regulation and cognitive processing (Bray, Stone, \& Gaskill, 2017).

In this paper, we present a synthesis of literature on equine-assisted psychotherapy (EAP) as unique addition to the portfolio of e trauma-informed interventions. Combining the therapeutic use of metaphor in client-directed settings such as play therapy (Boyd 
Webb, 2015; Landreth, 2002) with the unique experience of interacting with horses (Hayes, 2015; Yorke, Adams, \& Coady, 2008), EAP offers a promising approach to the treatment of trauma and stressor-related disorders. Unique to EAP is the inclusion of large prey animals in the therapeutic setting, inviting trauma survivors to interact with other sentient beings experiencing hypervigilance. The transparency and trust required of connection with the horses create an opportunity for survivors to experience safety in the therapeutic process.

\section{Animal-Assisted Therapy}

Animals have long been used to address issues of human suffering. Morrison's (2007) review of this history indicates that the practice has been around since the late 18th century when rabbits and chickens were included in the care of people with mental illness in England. Today animals are used to address an increasingly wide range of mental, behavioral and physical health conditions. Animal-assisted therapy (AAT) is the deliberate inclusion of a non-human animal in an intervention setting to enhance client outcomes (American Veterinary Medical Foundation [AVMA], 2017). Common interventions include the presence of dogs, rabbits (Pitheckoff, McLaughlin, \& de Medeiros, 2016), and guinea pigs (O'Haire, McKenzie, Beck, \& Slaughter, 2015) for outcomes such as stress reduction, reading fluency and the development of prosocial skills. Client populations include school-aged populations, older adults, incarcerated populations, individuals testifying in court cases, and college students. In these cases, the presence of the animal is theorized to provide a social buffer that maximizes the effect of the intervention (O'Haire et al., 2015). Children, for example, have been found to read more fluently when a dog is present (Jalongo, 2005). Horses are often included in animal-assisted activities and therapies, especially miniature breeds, for many of the same reasons (McCullough, RisleyCurtiss, \& Rorke, 2015). Full-size horses are included in some psychotherapeutic settings because of their distinction as large prey animals, specifically creating an environment that is not socially buffered. In their hypervigilant state, horses provide immediate, yet nonjudgmental, feedback to clients about their behavior (Hayes, 2015).

\section{The Evidence for Equine-Assisted Therapy}

The evidence for the impact that horses can have on therapeutic outcomes is limited due to small sample sizes and the lack of rigorous research designs. The results that have been published, however, are encouraging and worthy of consideration given the pressing need for alternative approaches to the treatment of trauma. A meta-analysis of equineassisted therapy for at-risk adolescents who had experienced trauma found a medium effect size for seven studies $(g=0.714, p<0.001)$ that all included pre- and post-intervention data (Wilkie, Germain, \& Theule, 2016). This effect reduced to a small/medium size ( $g=0.402$, $p=0.002$ ) when only the five studies that had both treatment and comparison groups were included. It is impressive that this effect is robust across the variations in treatment programs.

Systematic reviews of using horses in psychotherapy also indicate that interventions are promising for a range of populations (Kendall et al., 2015; Selby \& Smith-Osborne, 2013). Kendall et al. (2015) identified 15 studies that qualified for inclusion in their review. 
Only two of the studies (Bass, Duchowny, \& Llabre, 2009; Davis et al., 2009) were randomized-controlled trials. Kendall and colleagues (2015) report that although additional rigorously-designed studies were needed to conclude that equine-assisted interventions are efficacious, "equine-assisted interventions hold much promise, particularly in terms of child/adolescent social and behavioural issues" (p. 75). Selby and Smith-Osborne (2013) found a similarly small group of studies for review of equine-assisted therapy for populations with chronic illness or health conditions, noting that their analyses "lend credibility to the employment of equine-assisted techniques as an adjunct to traditional interventions for populations with health challenges" (p. 428).

Studies not included in the meta or systematic reviews of EAP include a qualitative study of adults in recovery from trauma, which identified the importance of the equinehuman bond given the horses' non-judgmental approach (Yorke et al., 2008). One participant in this qualitative study reflected on her experience, noting, "they [the horses] don't try and analyze you" (p. 23). Nurenberg and colleagues (2015) designed a randomlycontrolled trial using clinical incident reports and independent staff observations to compare both equine and canine-assisted therapy to standard treatment for 90 adults hospitalized for psychiatric conditions. Results indicate that the use of the EAGALAmodel EAP, delivered weekly for less than an hour and for no more than 10 sessions, was statistically more effective than canine therapy in reducing occurrence of violence in study participants. The authors posit that the differential effect of incorporating equines for psychiatric clients may be related to the fact that the horses are prey animals. "Nonpredatory equines, tending to mirror rather than direct human responses, may have a therapeutic advantage for some patients over more predatory species, such as canines and humans" (Nurenberg et al., p. 85). They further suggest that this status may have particular significance for clients who have experienced interpersonal trauma.

\section{Horses and Humans}

Humans have relied on horses for thousands of years, using them as a primary means of transportation on fields of battle and agriculture. In more recent years, horses became known for their ability to help people with both physical and emotional challenges. Today horses are used for a range of therapeutic activities including treatments for traumatic brain injury, autism spectrum disorders, sensory disorders, and stressor related disorders such as PTSD (Hayes, 2015).

What makes horses particularly suited to psychotherapeutic work is their distinction as prey animals (Hayes, 2015; Thomas \& Lytle, 2016). Horses live in a heightened state of awareness. This allows them to perceive outside stimuli with precision, keeping them, and their herd, safe. Horses pay close attention to even the most subtle shifts in congruency and mirror their behavior accordingly. When faced with an external threat, horses are often compelled to flee. They become closer and more cohesive as a group, protecting one another. Horses remain connected to the herd in times of stress. This is distinct from the individualistic model that humans often engage in and from the social isolation that trauma survivors can adopt. Observing equine behaviors provides humans the opportunity to think differently about the value of connectedness, especially in times of stress, anger, and fear as they process these emotions while interacting with the herd (Thomas \& Lytle, 2016). 
Horses' flight instinct is balanced with an innate curiosity and desire for connection. Horses are social, herd animals. They have distinct personalities and moods, just like people, and they have a hierarchical structure similar to that of families or the workplace. Like people, horses form special relationships with one another, give and accept affection, and set boundaries with one another (EAGALA, 2015).

Because the horses react and interact with humans consistent with their status as prey animals, clients receive feedback about their own behaviors in immediate, candid and nonjudgmental terms. The opportunity to observe how their behaviors - including attitudes, emotions, body language, and boundaries - affect the horses and to receive feedback in the moment is leveraged in equine-assisted interventions. The transparency of communication from prey animals living in a state of heightened awareness may paradoxically create a sense of safety in clients, especially those who have survived trauma, and allows space for nonjudgmental self-examination. With assistance from the treatment team, clients can become more open to examining their own post-traumatic stress responses. As Hayes (2015) states,

Many humans with certain types of emotional damage experience positive feelings of familiarity as they unconsciously identify with the two primary equine survival traits of hypervigilance and herd-dynamic-based social skills. These shared traits and interspecies identification can create mutual feelings of safety, acceptance, and compassion for both human and horse. In turn, this identification can lead a person to the self-awareness necessary for healing their emotional wounds. (p. 62)

EAP works with this mutuality to nurture the safe space initiated by the connection between clients and horses.

\section{The EAGALA Model of EAP}

While there are several emerging models of equine assisted psychotherapy, the EAGALA Model ${ }^{\circledR}$ offers a professional, standardized approach to engaging horses in treatment (Equine Assisted Growth and Learning Association [EAGALA], 2015). Founded in 1999 by L. Thomas, LCSW, EAGALA is a leading, international organization that supports the incorporation of horses into mental health and personal development work (EAGALA, 2010). Thomas recognized the efficacy of EAP as well as the need to professionalize standards for practice. She developed manuals, trainings, and a code of ethics to serve as scaffolding for this innovative therapeutic approach. With over 4,500 members in 50 countries and 700 active programs, EAGALA is the most widely known and respected equine-assisted psychotherapeutic model. EAGALA offers a fullydeveloped and professionally-endorsed treatment model for mental health professionals practicing EAP.

The EAGALA model is based in four tenets: 1) a team approach, 2) all sessions to be focused on the ground, 3) a solution-oriented belief system, and 4) an adherence to the EAGALA code of ethics. In each session, a treatment team is present. A licensed mental health professional and a certified equine specialist, along with horses and client(s), combine to form the treatment team. The mental health professional is charged with providing emotional safety for clients, treatment planning for each session, and ensuring 
ethical practice. The equine specialist provides physical safety for clients and horses, collaborates with the mental health professional to structure sessions, and offers observations of horse behavior. Working as a team, facilitators combine their individual expertise to provide an emotionally and physically safe experience for the client.

While therapeutic riding uses the kinesthetic connection between humans and horses to foster wellness, unmounted therapies primarily focus on the emotional connections that are present. EAGALA-model EAP is focused on the ground. None of the interactions between clients and horses include horsemanship or riding. Horsemanship develops horse skills, while EAP develops people skills. This greatly reduces the incidence of injury and enhances the accessibility of the treatment. Further adding to the accessibility of EAGALA as a treatment option is the fact that clients are not required to have any previous horse experience.

Usually, horses have no equipment on them during an EAGALA session. They are roaming freely in the space, allowing clients to meet them on equal footing rather than controlling them from a saddle - a powerful experience with a 1,200-pound animal. Because of the unmounted nature of the EAGALA model, clients are able to experiment through trial and error a multitude of ways for creating connection, developing relationships, and solving challenges. While clients can choose how close to get and where to touch the horses, the horses also have choices to move away or move closer. In order to interact with the horses, clients must experiment with different ways in which to connect.

The theoretical framework of the EAGALA-model is grounded in the use of selfdistancing through metaphor as the foundation for a solution-oriented approach. In the arena, the clients often find it easier to talk about what is happening with the horses in the moment than talking about their issues rather than reflectively and after the fact. The treatment team assumes that each client has solutions to their challenges and are capable of reaching their goals if given the opportunity to discover them. "We do not offer solutions, nor do we instruct the clients in how to interact with horses. We encourage them to develop their own methods, their own interpretations, and their own form of problem solving. This in turn enables them to take these new skills and apply them in the real world" (Thomas \& Lytle, 2016, p. 66). To be able to identify these solutions, EAGALA-model EAP allows clients the opportunity to shift from a self-immersed perspective to a selfdistanced perspective through the use of metaphor in interaction with the horses. "...People who self-distance focus less on recounting their experiences and more on reconstruing them in ways that provide insight and closure" (Kross \& Ayduk, 2011, p. 188). This happens within the context of trusting relationships with horses and humans often allowing clients the opportunity to gain some distance from their traumatic experiences before they can make meaning of them (Kross \& Ayduk, 2011).

Also setting this model apart from other equine models is its code of ethics. EAGALA's code of ethics creates a culture of professionalism for both mental health practitioners and equine specialists. Additionally, EAGALA has an ethics committee, providing rigorous and global enforcement of the code of ethics (EAGALA, 2015).

\section{Facilitating EAGALA-Model EAP}


Through a learned facilitation framework of reflective listening and observation known as SPUD'STM (EAGALA, 2015), the treatment team is able to create a therapeutic environment for a client's own story, perceptions, attitudes, emotions, and feelings to emerge.

The SPUD'S framework includes four criteria: Shifts, Patterns, Unique aspects, and Discrepancies ...(this) methodology (is) used by the team to track the activity and responses of both horse and client, although the primary focus is on the horses and symbols. As a client session progresses, the first four SPUD'S criteria allow the team to focus, pinpoint, and define moments of significance. The treatment team then takes these observations and reflects them back to the client in the form of question-asking, observational statements, metaphors, and invitations for clients to share their story. The four SPUD's, combined with these two actions - observing and reflecting - form the backbone of session execution. (Thomas \& Lytle, 2016, p. 71)

When people first enter the arena with the horses, they typically rely on their usual life coping skills to try to interact with or cajole the horses. If clients are unsure how to build rapport with the horse, they are often observed doing the same thing over and over again. Horses mirror that behavior back to the client through patterns of behavior. When the client shifts his or her awareness internally, such as an awareness that their prior actions were not working, the horses seamlessly shift their physical position, outward behaviors, and patterns. Thomas and Lytle (2016) explain that

Shifts in an EAGALA session pertain to any physical or behavioral change in the horses, other symbols, or the humans. Example of Shifts: The horses were together, but now they are apart. They were standing still, but now they are moving. The gate was open, and then it was closed. The client was outside along the wall but is now standing in the center of the arena. Every Shift in session, no matter how small, correlates to movement. Tracking movement is essential because movement indicates change. And change, in one form or another, is the global objective for both client and team. Shift by Shift, bit by bit, the client moves further from whatever state has him or her mired or stuck in place. (p. 72)

Unique to EAP is the focus on observable horse behavior using the SPUD'S framework. This model of intensive observation provides structure for the treatment team to stay within the model and resist interpreting client behavior, thus, allowing the client's own interpretations and solutions to come forth.

\section{Metaphors}

Metaphors reflecting trauma-related challenges arise frequently as clients seek to make meaning out of their experiences (McCormick \& McCormick, 1997; Pernicano, 2014, 2015). The interplay that occurs between the horses and clients often feels familiar. Clients describe their inner experience by relating it to what is happening externally in the moment. In an example from an actual session, the treatment team observed one horse putting its teeth on another horse's side repeatedly. The client exclaimed, "Why does he keep doing that to her?" The treatment team responded, "What is he doing to her?" The client said, 
"He's beating her up! Can you make it stop?" The client recognized a pattern with the horses and began to place her own narrative onto the experience. The treatment team probed, "What needs to happen to make him stop?" The client directed, "they need to separate." As the session progressed, the horse behavior shifted. The horse that had teeth on her sides and was being "beaten up" walked away from the other horse as the client approached to "separate them." The client said, "she left him but she needs protection." For a client with a traumatic abuse history, the concept of protection and safety are paramount to continue to explore in session with the horses. As this article expands upon later, the ability to create distance from the actual trauma through the experience with the horses allows the client to find new solutions for themselves as it relates to protection in an abusive situation.

The treatment team always follows the client's lead. The mental health professional assesses when and if the client is ready to further explore the metaphor verbally. Clients sometimes respond to the treatment team's questions about the horses with stories from their own lives using the metaphors as a bridge. Sometimes clients reveal histories of abuse and trauma for the first time in EAP sessions.

In the above-mentioned example, the one horse was being "beaten up" by the other horse and "needs protection." By her own description, this client's abuse history was playing out between the horses. In the EAGALA model, incorporating the interaction with the horses allows the metaphors to emerge by creating distance from the actual traumatic events that have occurred and allowing space for new interpretations (Thomas \& Lytle, 2016).

As the session continued, the client asserted that she and the treatment team needed to surround the horse to protect her and keep the other horse away. No matter how much the "abusive" horse tried to push his way into the circle of people, he was kept away from her. After several minutes, he walked away to the far end of the arena and stayed there for the remainder of the session. After a long pause looking in his direction, the client shared, "he's just like my ex-boyfriend."

Engaging with horses from a "self-distanced perspective" (Kross \& Ayduk, 2011, p. 188) allows clients to manage memories of their traumatic experiences that may emerge in sessions, while receiving a non-threatening, constant feedback system from sentient beings. "The ability to self-distance in the retelling of a negative experience or story allows storytellers to retain some form of objectivity in how they see that story....the third person allows a different perspective, shifting the client/storyteller from 'recounting/reliving' to reconstructing the story in a new way - a way that provides insight" (Thomas \& Lytle, 2016, p. 155).

The goal of EAGALA and other trauma-informed approaches is to move from problem-saturated narratives to more hopeful, solution-oriented narratives (Abels \& Abels, 2001). The theories informing these trauma-informed approaches include cognitive theory (Beck, 2011; Ledley, Marx, \& Heimberg, 2010), constructivist theory (Buckman, Kinney, \& Reese, 2008; Granvold, 2008) and neuroscience (Briere \& Lanktree, 2013; Germer, Siegel, \& Fulton, 2013). In EAP, the narratives, often in the form of metaphors, are at first constructed by the client (e.g., "He's beating her up! Can you make it stop?"). With 
reflective listening and feedback from the treatment team in a safe and affirming environment, the client then moves to changes in cognitive processing and more hopeful constructions of the horses' and personal experiences (e.g., "She left him but she needs protection."). These changes are facilitated at the neurobiological level as the limbic system is calmed through the establishment of trust and safety in a unique treatment setting. Even clients who experience human relationships as threatening due to prior experiences are able to engage safely with the horses and develop new healing pathways. The client can move past being "frozen" in prior trauma reactions and cognitively, neurologically, and somatically move to empowerment and hope.

\section{Presence}

Full presence is often a challenge for people who are experiencing trauma symptomatology. A "common denominator of all traumas is an alienation and disconnection from the body and a reduced capacity to be present in the here and now" (Emerson \& Hopper, 2011, p. xi). Disembodiment is a powerful coping mechanism, yet one that does not easily allow engagement in the therapeutic process. Although clients can feel initially anxious about interacting with horses in an EAGALA session, their perspective often shifts to feeling safe in the presence of a nonjudgmental being. The horses provide honest feedback free of ego, bias, or agenda, allowing individuals to be more receptive to the exploration and acceptance of the sensation. These feelings of safety and sensation are scaffolded by the rhythms of tail swishing, ear movements, hoofbeats and expressive exhales. Once clients can experience safety and explore their metaphors and symbols with the horses, they are better equipped to process their emotions and make meaning out of their experiences in an emotionally safe manner (Kross \& Ayduk, 2011).

\section{Meeting Standards}

Although EAP does not yet meet the gold standard for an evidence-based practice, it meets the SAMSHA key principles for a trauma-informed approach. Being a relatively new field, outcome studies were not published until 2008, thus only one meta-analysis has been conducted to date (Wilkie et al., 2016). The emerging nature of this field has also limited the amount of targeted funding available to researchers often needed to conduct randomized-controlled trials. The vast majority of public and private funding on animalassisted interactions has gone to studies including canines. Early indications, however, are that EAP holds promise for work with at-risk populations (Wilkie et al., 2016). Additional, rigorously-designed studies are needed to further explore the potential of EAP.

The EAGALA model of EAP meets the Six Key Principles of a Trauma-informed Approach (SAMHSA, 2014). The key principles, as mentioned previously include: 1) safety, 2) trustworthiness and transparency, 3) peer support, 4) collaboration and mutuality, 5) empowerment, voice, and choice, and 6) cultural, historical, and gender issues. EAGALA's Code of Ethics (EAGALA, 2015) directly links to these key principles. Safety is paramount. Trustworthiness and transparency are critical aspects of the EAGALA model. Peer support can include either working with those with lived experience or in regard to children and adolescents, non-offending parents or caregivers who can give validation and voice to the children's experiences. In EAGALA, one can work in groups 
of those with lived experiences or in the case of individual work, one can argue that the horses provide peer support, collaboration and mutuality - affirmation in the most basic and pure ways. The EAGALA model is clearly client-directed, promoting empowerment, voice, and choice. Finally, the EAGALA Code of Ethics promotes practice that is sensitive to cultural, historical and gender issues. "The ethics code is based on the fundamental values of overall safety and well-being of clients, foremost above all other considerations...it is our quest to build the emerging field of Equine Assisted Psychotherapy as a valid, professional, safe, and respected instrument for growth and learning" (EAGALA, 2015, p. 20).

Further supporting the claim that EAP is well-suited to trauma work is its synchronicity with Peter Levine's work. Levine (2010), a leading trauma therapist, developed steps that can help a person move from alienation and disconnection from their body to healing through somatic experiencing. These include establishing an environment of relative safety, supporting initial exploration and acceptance of sensation, and establishing pendulation and containment - the innate power of rhythm. EAP provides each of these. SAMHSA has been leading the way in promoting trauma-informed care in the U.S., acknowledging that both recovery and resilience are key outcomes for in both individual and family work. The EAGALA®-model of EAP promotes these same tenets through its Code of Ethics, requiring clinicians to utilize empowering methods that provide voice and choice to those who have had none.

\section{Limitations}

One clear limitation of EAP is accessibility in terms of both location and cost. Horses tend to live in barns and on land that can be inaccessible to public transportation, often requiring clients to have private transportation. This can be a significant barrier to access for clients in urban areas. Further, the cost of maintaining a herd of horses and compensating two members of the treatment team is not insignificant. While some mental health professionals do accept insurance, the reimbursement rate is typically not enough to cover the entire session. Some organizations, such as Gateway HorseWorks of Malvern, Pennsylvania, have worked to reduce this barrier by operating as a non-profit and securing grants from local and national organizations (Gateway HorseWorks, 2017).

Social workers are encouraged to learn more about EAP by connecting with local treatment teams including equine specialists and behavioral health professionals, receiving training and attending demonstrations. EAGALA-certified mental health professionals and equine specialists are often willing to meet with behavioral health professionals and discuss their work.

The evidence base for EAP is limited due to both historical and practical reasons. Social workers also need to support the building of an evidence base by engaging in research, serving as liaisons between university research teams and EAGALA teams, and referring research participants to ongoing studies. Specifically, there is a strong need for randomized controlled trials that can isolate both the active elements of the treatment and shed light on the mechanisms of change. These studies then need to be replicated with varied populations with varied presenting issues. Animal-assisted therapy, with horses or 
other animals, is not for everyone, nor is it for every condition. More research is needed to identify those populations and those conditions for which it is most effective.

\section{Next Steps}

As the primary providers of mental and behavioral health services in the United States, social workers are keenly aware of the need for a portfolio of treatment methods to manage the increasing demand for services. Vulnerable clients who have experienced trauma deserve to have an array of treatment options available - in addition to or instead of traditional talk therapies. Talk therapies are simply not efficacious for many who are struggling with the impact of trauma, who are alienated and disconnected from their bodies and unable to verbally process a myriad of sensations (Levine, 2010). As Kazdin and Blaze (2011) suggest, "interventions that vary widely in their reach, focus, costs, effects, and other dimensions are crucial" (p. 33), including experiential and expressive therapies such as EAP.

The ethical call to meet the needs of vulnerable populations is loud. Social workers are poised to take a lead role in promoting a robust, accessible and inclusive approach to the delivery of mental and behavioral health care services. With a core mission of enhancing human well-being and meeting the basic human needs of all people, the role of promoting the use of and research on interventions such as EAP is a natural fit. The EAGALA model of EAP is a particularly good fit with social work. Perhaps most important is the core belief that people have the inherent capacity to recover and be resilient; they already have solutions to the challenges they face and can access them when provided the appropriate space to do so. This is evidenced by the way in which EAGALA supports client-directed work.

Another key congruence between social work and EAGALA is the way in which the treatment team allows individuals to be the experts in the arena, explicitly resisting a controlling role. EAGALA also challenges the assumptions of talk therapy by privileging the power of nonverbal and nonhuman communication. This explicit shift in power dynamics supports social work's anti-oppressive and empowerment approach, allowing subjugated knowledge to emerge in treatment (Dominelli, 2002; Hartman, 1992). The American Counseling Association (2016) has already adopted practice competencies for animal assisted therapies. It is time for social work to join this movement and specifically consider the ways in which EAGALA might be an appropriate treatment for some clients.

\section{References}

Abels, S., \& Abels, P. (2001). Understanding narrative therapy: A guidebook for the social worker. New York: Springer.

American Counseling Association. (2016). Animal-assisted therapy in counseling competencies. Retrieved from: www.counseling.org/knowledge-center/competencies

American Red Cross. (2012). Disaster mental health handbook: Disaster services. Washington DC: Author.

American Veterinary Medical Foundation [AVMA]. (2017). Animal-assisted 
interventions: Definitions. Retrieved on June 5, 2017, from

https://www.avma.org/KB/Policies/Pages/Animal-Assisted-Interventions-

Definitions.aspx

Anda, R. F., Felitti, V. J., Bremner, J. D., Walker, J. D., Whitfield, C. H., Perry, B. D., ... Giles, W. H. (2006). The enduring effects of abuse and related adverse experiences in childhood. European Archives of Psychiatry and Clinical Neuroscience, 256(3), 174186. doi: https://doi.org/10.1007/s00406-005-0624-4

Anda, R., Felitti, V. J., \& Corwin, D. L. (2014). Adverse Childhood Experiences and long-term health. ACEs: Informing Best Practice, AVA/NHCVA, Section, 1.

Baker, L. M., Williams, L. M., Korgaonkar, M. S., Cohen, R. A., Heaps, J. M., \& Paul, R. H. (2013). Impact of early vs. late childhood early life stress on brain morphometrics. Brain Imaging and Behavior, 7(2), 196-203. doi: https://doi.org/10.1007/s11682-012-9215-y

Bass, M. M., Duchowny, C. A., \& Llabre, M. M. (2009). The effect of therapeutic horseback riding on social functioning in children with autism. Journal of Autism and Developmental Disorders, 39, 1261-1267. doi: https://doi.org/10.1007/s10803-009$\underline{0734-3}$

Beck, J. S. (2011). Cognitive behavior therapy: Basics and beyond ( $2^{\text {nd }}$ ed.) New York: Guilford.

Bourne, C., Mackay, C. E., \& Holmes, E. A. (2013). The neural basis of flashback formation: The impact of viewing trauma. Psychological medicine, 43(7), 1521-1532. doi: https://doi.org/10.1017/S0033291712002358

Boyd Webb, N. (2015). Play therapy with children and adolescents in crisis (4th ed.). New York: Guilford.

Bray, S. S., Stone, J. P., \& Gaskill, R. L. (2017). The impact of trauma on brain development: A neurodevelopmentally appropriate model for play therapists. In R. L. Steen (Ed.), Emerging research in play therapy, child counseling, and consultation (pp. 20-39). Hershey, PA: IGI Global. doi: https://doi.org/10.4018/978-1-5225-22249.ch002

Briere, J. N., \& Lanktree, C. B. (2013). Treating complex trauma in adolescents and young adults. Thousand Oaks, CA: Sage.

Buckman, R., Kinney, D., \& Reese, A. (2008). Narrative therapies. In N. Coady \& P. Lehman (Eds.), Theoretical perspectives for direct social work practice, (pp. 369400). New York: Springer.

Cronholm, P. F., Forke, C. M., Wade, R., Bair-Merritt, M. H., Davis, M., HarkinsSchwarz, M., ...Fein, J. A. (2015). Adverse childhood experiences: Expanding the concept of adversity. American Journal of Preventive Medicine, 49(3), 354-361. doi: https://doi.org/10.1016/j.amepre.2015.02.001

Dannlowski, U., Stuhrmann, A., Beutelmann, V., Zwanzger, P., Lenzen, T., Grotegerd, 
D., ...Lindner, C. (2012). Limbic scars: Long-term consequences of childhood maltreatment revealed by functional and structural magnetic resonance imaging. Biological Psychiatry, 71(4), 286-293. doi: https://doi.org/10.1016/j.biopsych.2011.10.021

Davis, E., Davies, B., Wolfe, R., Raadsveld, R., Heine, B., Thomason, P., ...Graham, H. K. (2009). A randomized controlled trial of the impact of therapeutic horse riding on the quality of life, health, and function of children with cerebral palsy.

Developmental Medicine and Child Neurology, 51, 111-119. doi: https://doi.org/10.1111/j.1469-8749.2008.03245.x

Davis, D. M., \& Hayes, J. A. (2011). What are the benefits of mindfulness? A practice review of psychotherapy-related research. Psychotherapy, 48(2), 198-208. doi: https://doi.org/10.1037/a0022062

Dominelli, L. (2002). Anti-oppressive social work theory and practice. New York: Palgrave Macmillan. doi: https://doi.org/10.1007/978-1-4039-1400-2

Equine Assisted Growth and Learning Association [EAGALA]. (2010). Welcome. EAGALA Website. Retrieved from www.eagala.org

EAGALA. (2015). Fundamentals of the EAGALA model: Training manual (8th ed.). Santaquin, UT: Author.

Emerson, D., \& Hopper, E. (2011). Overcoming trauma through yoga: Reclaiming your body. Berkeley, CA: North Atlantic Books.

Felitti, V. J., Anda, R. F., Nordenberg, D., Williamson, D. F., Spitz, A. M., Edwards, V., Koss, M. P., \& Marks, J. S. (1998). Relationship of childhood abuse and household dysfunction to many of the leading causes of death in adults: The Adverse Childhood Experiences (ACE) study. American Journal of Preventative Medicine, 14, 245-258. doi: https://doi.org/10.1016/S0749-3797(98)00017-8

Gateway HorseWorks. (2017). Our mission. Retrieved from http://www.gatewayhorseworks.org

Germer, C. K., Siegel, R. D., \& Fulton, P. R. (2013). Mindfulness and psychotherapy (2nd ed.) New York: Guilford.

Getz, L. (2012). Trauma-focused cognitive behavioral therapy: Hope for abused children. Social Work Today, 12, 22-24.

Granvold, D. K. (2008). Constructivist theory. In N. Coady \& P. Lehmann (Eds.), Theoretical perspectives for direct social work practice (pp. 401-428). New York: Springer.

Hartman, A. (1992). In search of subjugated knowledge. Social Work, 37, 483-484.

Hayes, T. (2015). Riding home: The power of horses to heal. NY: St. Martin's Press.

Jalongo, M. R. (2005). What are all these dogs doing at school? Using therapy dogs to promote children's reading practice. Childhood Education, 81(3), 152-158. doi: 


\section{https://doi.org/10.1007/s10643-009-0349-5}

Kazdin, A. E., \& Blaze, S. L. (2011). Rebooting psychotherapy research and practice to reduce the burden of mental illness. Perspectives on Psychological Science, 6(1), 2137. doi: https://doi.org/10.1177/1745691610393527

Kendall, E., Maujean, A., Pepping, C.A., Downes, M., Lakhani, A., Byrne, J., \& Macfarlane, K. (2015). A systematic review of the efficacy of equine-assisted interventions on psychological outcomes. European Journal of Psychotherapy \& Counselling, 17, 57-79. doi: https://doi.org/10.1080/13642537.2014.996169

Kliethermes, M., \& Wamser, R. (2012). Adolescents with complex trauma. In J. A. Cohen, A. P. Mannarino, \& E. Deblinger, E. (Eds.), Trauma-focused CBT for children and adolescents: Treatment applications (pp 175-196). New York: Guilford.

Kross, E., \& Ayduk, O. (2011). Making meaning out of negative experiences by selfdistancing. Current Directions in Psychological Science, 20, 187-191. doi: https://doi.org/10.1177/0963721411408883

Landreth, G. L. (2002). Therapeutic limit setting in the play therapy relationship. Professional Psychology: Research and Practice, 33(6), 529-535. doi: https://doi.org/10.1037/0735-7028.33.6.529

Larkin, H., Shields, J. J., \& Anda, R. F. (2012). The health and social consequences of Adverse Childhood Experiences (ACE) across the lifespan: An introduction to prevention and intervention in the community. Journal of Prevention \& Intervention in the Community, 40(4), 263-270. doi: https://doi.org/10.1080/10852352.2012.70743

Ledley, D. R., Marx, B. P., \& Heimberg, R. G. (2010). Making cognitive-behavioral therapy work: Clinical process for new practitioners (2nd ed.). New York: Guilford.

Levine, P. A. (2010). In an unspoken voice: How the body releases trauma and restores goodness. Berkeley, CA: North Atlantic Books.

McCormick, A. R., \& McCormick, M. D. (1997). Horse sense and the human heart: What horses can teach us about trust, bonding, creativity, and spirituality. Deerfield Beach, FL: Health Communications, Inc.

McCullough, L., Risley-Curtiss, C., \& Rorke, J. (2015). Equine facilitated psychotherapy: A pilot study of effect on posttraumatic stress symptoms in maltreated youth. Journal of Infant, Child, and Adolescent Psychotherapy, 14, 158173. doi: https://doi.org/10.1080/15289168.2015.1021658

Morrison, M. L. (2007). Health benefits of animal-assisted interventions. Complementary Health Practice Review, 12, 51-62. doi: https://doi.org/10.1177/1533210107302397

Murphy, A., Steele, H., Steele, M., Allman, B., Kastner, T., \& Dube, S. R. (2016). The clinical Adverse Childhood Experiences (ACEs) questionnaire: Implications for trauma-informed behavioral healthcare. In R. D. Briggs (Ed.), Integrated early childhood behavioral health in primary care (pp. 7-16). Switzerland: Springer International Publishing. doi: https://doi.org/10.1007/978-3-319-31815-8_2 
Nurenberg, J. R., Schleifer, S. J., Shaffer, T. M., Yellin, M., Desai, P. J., Amin, R., ...Montalvo, C. (2015). Animal-assisted therapy with chronic psychiatric inpatients: Equine-assisted psychotherapy and aggressive behavior. Psychiatric Services, 66(1), 80-86. doi: https://doi.org/10.1176/appi.ps.201300524

O'Haire, M. E., McKenzie, S. J., Beck, A. M., \& Slaughter, V. (2015). Animals may act as social buffers: Skin conductance arousal in children with autism spectrum disorder in a social context. Developmental Psychobiology, 57(5), 584-595. doi: https://doi.org/10.1002/dev.21310

Omidi, A. (2013). Towards an integrative approach to trauma study. Archives of Trauma Research, 2(1), 1-2. doi: https://doi.org/10.5812/atr.11288

Pernicano, P. (2014). Using trauma-focused therapy stories: Interventions for therapists, children and their caregivers. New York: Routledge.

Pernicano, P. (2015). Metaphors and stories in play therapy. In C. Schaefer, K.

O'Connor, \& L. Braverman (Eds.), Handbook of play therapy (2nd ed., pp. 259-275). New York: Wiley. doi: https://doi.org/10.1002/9781119140467.ch12

Pitheckoff, N., McLaughlin, S. J., \& de Medeiros, K. (2016). "Calm . . satisfied. . . comforting": The experience and meaning of rabbit-assisted activities for older adults. Journal of Applied Gerontology, online, 1-12. doi: https://doi.org/10.1177/0733464816680322

Rhodes, A. M. (2015). Claiming peaceful embodiment through yoga in the aftermath of trauma. Complementary Therapies in Clinical Practice, 21, 247-256. doi: https://doi.org/10.1016/j.ctcp.2015.09.004

Sekiguchi, A., Sugiura, M., Taki, Y., Kotozaki, Y., Nouchi, R., Takeuchi, H., ...Sakuma, A. (2013). Brain structural changes as vulnerability factors and acquired signs of post-earthquake stress. Molecular Psychiatry, 18(5), 618-623. doi: https://doi.org/10.1038/mp.2012.51

Selby, A., \& Smith-Osborne, A. (2013). A systematic review of effectiveness of complementary and adjunct therapies and interventions involving equines. Health Psychology, 32, 418-432. doi: https://doi.org/10.1037/a0029188

Substance Abuse and Mental Health Services Administration [SAMSHA]. (2014). SAMHSA's concept of trauma and guidance for a trauma-informed approach. HHS Publication No. (SMA) 14-4884. Rockville, MD: Substance Abuse and Mental Health Services Administration.

Thomas, L., \& Lytle, M. (2016). Transforming therapy through horses. Santaquin, UT: EAGALA.

van der Kolk, B. A. (2002). Beyond the talking cure: Somatic experience and subcortical imprints in the treatment of trauma. In F. Shapiro (Ed.), EMDR as an integrative psychotherapy approach: Experts of diverse orientations explore the paradigm prism (Vol. vii, pp. 57-83). Washington, DC: American Psychological Association. doi: https://doi.org/10.1037/10512-003 
van der Kolk, B. A. (2014). The body keeps the score: Brain, mind, and body in the healing of trauma. London, UK: Penguin Books.

van der Kolk, B. A., \& McFarlane, A. C. (Eds.). (2012). Traumatic stress: The effects of overwhelming experience on mind, body, and society. NY: Guilford.

van der Kolk, B. A., Stone, L., West, J., Rhodes, A., Emerson, D., Suvak, M., \& Spinazzola, J. (2014). Yoga as an adjunctive treatment for posttraumatic stress disorder: A randomized clinical trial. Journal of Clinical Psychiatry, 75, e1-e7. doi: https://doi.org/10.4088/JCP.13m08561

West, J., Lian, B., \& Spinazzola, J. (2016). Trauma sensitive yoga as complementary treatment for posttraumatic stress disorder: A qualitative descriptive analysis. International Journal of Stress Management, 24(2), 173-195. doi: http://dx.doi.org/10.1037/str0000040

Wilkie, K. D., Germain, S., \& Theule, J. (2016). Evaluating the efficacy of equine therapy among at-risk youth: A meta-analysis. Anthrozoös, 29(3), 377-393. doi: https://doi.org/10.1080/08927936.2016.1189747

Yorke, J., Adams, C., \& Coady, N. (2008). Therapeutic value of equine-human bonding in recovery from trauma. Anthrozoos, 21(1), 17-30. doi: https://doi.org/10.2752/089279308X274038

Zucker, M., Spinazzola, J., Pollack, A. A., Pepe, L., \& Barry, S. (2010). Getting teachers in on the act: Evaluation of a theater- and classroom-based youth violence prevention program. Journal of School Violence, 9, 117-135. doi: https://doi.org/10.1080/15388220903479628

Author note: Address correspondence to: Dr. Page Walker Buck, LSW, Associate Professor, Department of Graduate Social Work, West Chester University, West Chester, PA, 19383.610-430-4171, pbuck@wcupa.edu. 